\title{
Deterministic Sampling-Based Motion Planning: Optimality, Complexity, and Performance
}

\author{
Lucas Janson \\ Department of Statistics, Stanford University, USA
}

Brian Ichter

Department of Aeronautics and Astronautics, Stanford University, USA

\author{
Marco Pavone* \\ Department of Aeronautics and Astronautics, Stanford University, USA
}

\begin{abstract}
Probabilistic sampling-based algorithms, such as the probabilistic roadmap (PRM) and the rapidly-exploring random tree (RRT) algorithms, represent one of the most successful approaches to robotic motion planning, due to their strong theoretical properties (in terms of probabilistic completeness or even asymptotic optimality) and remarkable practical performance. Such algorithms are probabilistic in that they compute a path by connecting independently and identically distributed (i.i.d.) random points in the configuration space. Their randomization aspect, however, makes several tasks challenging, including certification for safety-critical applications and use of offline computation to improve real-time execution. Hence, an important open question is whether similar (or better) theoretical guarantees and practical performance could be obtained by considering deterministic, as opposed to random sampling sequences. The objective of this paper is to provide a rigorous answer to this question. Specifically, we first show that PRM, for a certain selection of tuning parameters and deterministic low-dispersion sampling sequences, is deterministically asymptotically optimal, i.e., it returns a path whose cost converges deterministically to the optimal one as the number of points goes to infinity. Second, we characterize the convergence rate, and we find that the factor of sub-optimality can be very explicitly upper-bounded in terms of the $\ell_{2}$-dispersion of the sampling sequence and the connection radius of PRM. Third, we show that an asymptotically optimal version of PRM exists with computational and space complexity arbitrarily close to $O(n)$ (the theoretical lower bound), where $n$ is the number of points in the sequence. This is in stark contrast to the $O(n \log n)$ complexity results for existing asymptoticallyoptimal probabilistic planners. Fourth, we show that our theoretical results and insights extend to other batch-processing
\end{abstract}

\footnotetext{
* Corresponding author; email: pavone@ stanford.edu

This work was originally presented at the 17th International Symposium on Robotics Research, ISRR 2015. This extended and revised version includes a novel section on extensions of this work to $k$-nearest-neighbor algorithms, non-PRM algorithms, non-lattice sampling, non-uniform sampling, and kinodynamic motion planning. It also includes a significantly extended simulation section.
} 
algorithms such as FMT $^{*}$, to non-uniform sampling strategies, to $k$-nearest-neighbor implementations, and to differentiallyconstrained problems. Finally, through numerical experiments, we show that planning with deterministic low-dispersion sampling generally provides superior performance in terms of path cost and success rate.

\section{Introduction}

Probabilistic sampling-based algorithms represent a particularly successful approach to robotic motion planning problems [Thrun et al., 2005, Lavalle, 2006]. The key idea behind probabilistic sampling-based algorithms is to avoid the explicit construction of the configuration space (which can be prohibitive in complex planning problems) and instead conduct a search that probabilistically probes the configuration space with independently and identically distributed (i.i.d.) random samples. This probing is enabled by a collision detection module, which the motion planning algorithm considers as a "black box" [Lavalle, 2006]. Examples, roughly in chronological order, include the probabilistic roadmap algorithm (PRM) [Kavraki et al., 1996], expansive space trees (EST) [Hsu et al. 1999, Phillips et al., 2004], Lazy-PRM [Bohlin and Kavraki. 2000], the rapidly exploring random trees algorithm (RRT) [LaValle and Kuffner. 2001], sampling-based roadmap of trees (SRT) [Plaku et al., 2005], rapidly-exploring roadmap [Alterovitz et al., 2011], PRM* and RRT* [Karaman and Frazzoli 2011], RRT [Arslan and Tsiotras, 2013, and the fast marching tree algorithm (FMT*) Janson et al. 2015]. A central result is that these algorithms provide probabilistic completeness guarantees in the sense that the probability that the planner fails to return a solution, if one exists, decays to zero as the number of samples approaches infinity [Barraquand et al. 2000]. Recently, it has been proven that RRT*, PRM*, RRT\#, and FMT* are asymptotically optimal, i.e., the cost of the returned solution converges almost surely to the optimum [Karaman and Frazzoli, 2011, Arslan and Tsiotras, 2013, Janson et al., 2015].

It is natural to wonder whether the theoretical guarantees and practical performance of sampling-based algorithms would hold if these algorithms were to be de-randomized, i.e., run on a deterministic, as opposed to random sampling sequence. This is an important question, as de-randomized planners would significantly simplify the certification process (as needed for safety-critical applications), enable the use of offline computation (particularly important for planning under differential constraints or in high-dimensional spaces - exactly the regime for which sampling-based planners are designed), and, in the case of lattice sequences, drastically simplify a number of operations (e.g., locating nearby samples). This question has received relatively little attention in the literature. Specifically, previous research [Branicky et al. 2001, LaValle et al. 2004. Hsu et al. 2006] has focused on the performance of de-randomized versions of sampling-based planners in terms of convergence to feasible paths. A number of deterministic variants of the PRM algorithm were shown to be resolution complete (i.e., provably converging to a feasible solution as $n \rightarrow \infty$ ) and, perhaps surprisingly, offer superior performance on an extensive set of numerical experiments [Branicky et al., 2001, LaValle et al., 2004]. Prompted by these results, a number of deterministic low-dispersion, incremental sequences have been specifically tailored to motion planning problems [Yershova and LaValle, 2004, Lindemann et al., 2005, Yershova et al., 2010].

The results in [Branicky et al., 2001, LaValle et al., 2004, Hsu et al., 2006] are restricted to convergence to feasible, as opposed to optimal paths. Several questions are still open. Are there advantages of i.i.d. sampling in terms of convergence to an optimal path? Can convergence rate guarantees for the case of deterministic sampling be provided, similar to what is done for probabilistic planners in [Janson et al., 2015, Dobson et al., 2015]? For a given number of samples, are there advantages in terms of computational and space complexity? The objective of this paper is to rigorously address these questions. Our focus is on the PRM algorithm. However, we show that similar results hold for many of the existing batch (i.e., not anytime) algorithms, including Lazy-PRM and FMT* .

Statement of Contributions: The contributions of this paper are as follows.

Deterministic asymptotic optimality of sampling-based planning: We show that the PRM algorithm is asymptotically optimal when run on deterministic sampling sequences in $d$ dimensions whose $\ell_{2}$-dispersion is upper-bounded by 
$\gamma n^{-1 / d}$, for some $\gamma \in \mathbb{R}_{>0}$ (we refer to such sequences as deterministic low-dispersion sequences), and with a connection radius $r_{n} \in \omega\left(n^{-1 / d}\right)$ In other words, the cost of the solution computed over $n$ samples converges deterministically to the optimum as $n \rightarrow \infty$. As a comparison, the analogue result for the case of i.i.d. random sampling holds almost surely or in probability [Karaman and Frazzoli, 2011, Janson et al. 2015] (as opposed to deterministically) and requires a connection radius $\Omega\left((\log (n) / n)^{1 / d}\right)$, i.e., bigger.

Convergence rate: We show that, in the absence of obstacles, the factor of sub-optimality of PRM is upper-bounded by $2 D_{n} /\left(r_{n}-2 D_{n}\right)$, where $D_{n}$ is the $\ell_{2}$-dispersion of the sampling sequence. A slightly more sophisticated result holds for the obstacle-cluttered case. As a comparison, the analogue result for the case of i.i.d. sampling only holds in probability and is much more involved (and less interpretable) [Janson et al., 2015]. Our results could be instrumental to the certification of sampling-based planners.

Computational and space complexity: We prove that PRM, when run on a low-dispersion lattice, has computational and space complexity $O\left(n^{2} r_{n}^{d}\right)$. As asymptotic optimality can be obtained using $r_{n} \in \omega\left(n^{-1 / d}\right)$, there exists an asymptotically optimal version of PRM with computational and space complexity $\omega(n)$, where $O(n)$ represents the theoretical lower bound (as, at the very least, $n$ operations need to be carried out to load samples into memory). As a comparison, the analogous complexity results for the case of i.i.d. sampling are of order $O(n \log (n))$ [Karaman and Frazzoli, 2011].

Extensions: We extend the contributions in all three of the preceding categories to much broader settings. Specifically, we find that many of the results that hold for PRM run on a low-dispersion lattice hold either exactly or approximately for $k$-nearest-neighbor algorithms, for other batch-processing algorithms such as $\mathrm{FMT}^{*}$, for non-lattice low-dispersion sampling such as the Halton sequence, for non-uniform sampling, and for kinodynamic planning.

Experimental performance: Finally, we compare performance (in terms of path cost and success rates) of deterministic low-dispersion sampling versus i.i.d. sampling on a variety of test cases ranging from two to eight dimensions and including geometric, kinematic chain, and kinodynamic planning problems. In all our examples, for a given number of samples, deterministic low-dispersion sampling performs no worse and sometimes substantially better than i.i.d. sampling (this is not even accounting for the potential significant speed-ups in runtime, e.g., due to fast nearest-neighbor indexing).

The key insight behind our theoretical results (e.g., smaller required connection radius, better complexity, etc.) is the factor difference in dispersion between deterministic low-dispersion sequences versus i.i.d. sequences, namely $O\left(n^{-1 / d}\right)$ versus $O\left((\log n)^{1 / d} n^{-1 / d}\right)$ [Deheuvels, 1983, Niederreiter, 1992]. Interestingly, the same $O\left(n^{-1 / d}\right)$ dispersion can be achieved with non-i.i.d. random sequences, e.g., randomly rotated and offset lattices. As we will show, these sequences enjoy the same deterministic performance guarantees of deterministic low-dispersion sequences and retain many of the benefits of deterministic sampling (e.g., fast nearest-neighbor indexing). Additionally, their "controlled" randomness may allow them to address some potential issues with deterministic sequences (in particular lattices), e.g., avoiding axisalignment issues in which entire rows of samples may become infeasible due to alignment along an obstacle boundary. In this perspective, achieving deterministic guarantees is really a matter of i.i.d. sampling versus non-i.i.d. low-dispersion sampling (with deterministic sampling as a prominent case), as opposed to random versus deterministic. Collectively, our results, complementing and corroborating those in [Branicky et al., 2001, LaValle et al., 2004], strongly suggest that both the study and application of sampling-based algorithms should adopt non-i.i.d. low-dispersion sampling. From a different viewpoint, our results provide a theoretical bridge between sampling-based algorithms with i.i.d. sampling and

\footnotetext{
${ }^{1}$ For $f, g: \mathbb{N} \rightarrow \mathbb{R}$, we say $f \in O(g)$ if there exists $n_{0} \in \mathbb{N}$ and $k \in \mathbb{R}_{>0}$ such that $|f(n)| \leq k|g(n)|$ for all $n \geq n_{0}$. We say $f \in \Omega(g)$ if there exists $n_{0} \in \mathbb{N}$ and $k \in \mathbb{R}_{>0}$ such that $|f(n)| \geq k|g(n)|$ for all $n \geq n_{0}$. Finally, we say $f \in \omega(\bar{g})$ if $\lim _{n \rightarrow \infty} f(n) / g(n)=\infty$.
} 
non-sampling-based algorithms on regular grids (e.g., D* [Stentz, 1995] and related kinodynamic variants [Pivtoraiko et al. 2009|).

Organization: This paper is structured as follows. In Section 2 we provide a review of known concepts from lowdispersion sampling, with a focus on $\ell_{2}$-dispersion. In Section 3 we formally define the optimal path planning problem. In Section 4 we present our three main theoretical results for planning with low-dispersion sequences: asymptotic optimality, convergence rate, and computational and space complexity. In Section 5 we extend the results from Section 4 to other batchprocessing algorithms, non-uniform sampling, and kinodynamic motion planning. In Section 6 we present results from numerical experiments supporting our statements. Finally, in Section 7 we draw some conclusions and discuss directions for future work.

\section{Background}

A key characteristic of any set of points on a finite domain is its $\ell_{2}$-dispersion. This concept will be particularly useful in elucidating the advantages of deterministic sampling over i.i.d. sampling. As such, in this section we review some relevant properties and results on the $\ell_{2}$-dispersion.

Definition 1 ( $\ell_{2}$-dispersion). For a finite, nonempty set $S$ of points contained in a d-dimensional compact Euclidean subspace $\mathcal{X}$ with positive Lebesgue measure, its $\ell_{2}$-dispersion $D(S)$ is defined as

$$
D(S):=\sup _{x \in \mathcal{X}} \min _{s \in S}\|s-x\|_{2}=\sup \{r>0: \exists x \in \mathcal{X} \text { with } B(x, r) \cap S=\emptyset\}
$$

where $B(x, r)$ is the open ball of radius $r$ centered at $x$.

Intuitively, the $\ell_{2}$-dispersion quantifies how well a space is covered by a set of points $S$ in terms of the largest open Euclidean ball that touches none of the points. The quantity $D(S)$ is important in the analysis of path optimality as an optimal path may pass through an empty ball of radius $D(S)$. Hence, $D(S)$ bounds how closely any path tracing through points in $S$ can possibly approximate that optimal path.

The $\ell_{2}$-dispersion of a set of deterministic or random points is often hard to compute, but luckily it can be bounded by the more-analytically-tractable $\ell_{\infty}$-dispersion. The $\ell_{\infty}$-dispersion is defined by simply replacing the $\ell_{2}$-norm in equation (1) by the $\ell_{\infty}$-norm, or max-norm. The $\ell_{\infty}$-dispersion of a set $S$, which we will denote by $D_{\infty}(S)$, is related to the $\ell_{2}$-dispersion in $d$ dimensions by [Niederreiter. 1992],

$$
D_{\infty}(S) \leq D(S) \leq \sqrt{d} D_{\infty}(S)
$$

which allows us to bound $D(S)$ when $D_{\infty}(S)$ is easier to compute. In particular, an important result due to [Deheuvels, 1983 is that the $\ell_{\infty}$-dispersion of $n$ independent uniformly sampled points on $[0,1]^{d}$ is $O\left((\log (n) / n)^{1 / d}\right)$ with probability 1. Corollary to this is that the $\ell_{2}$-dispersion is also $O\left((\log (n) / n)^{1 / d}\right)$ with probability 1 .

Remarkably, there are deterministic sequences with $\ell_{2}$-dispersions of order $O\left(n^{-1 / d}\right)$, an improvement by a factor $\log (n)^{1 / d}$. (Strictly speaking, one should distinguish point sets, where the number of points is specified in advance, from sequences [LaValle et al., 2004]—in this paper we will simply refer to both as "sequences.") For instance, the Sukharev sequence [Sukharev, 1971], whereby $[0,1]^{d}$ is gridded into $n=k^{d}$ hypercubes and their centers are taken as the sampled points, can easily be shown to have $\ell_{2}$-dispersion of $(\sqrt{d} / 2) n^{-1 / d}$ for $n=k^{d}$ points. As we will see in Section 4 , the use of sample sequences with lower $\ell_{2}$-dispersions confers on PRM a number of beneficial properties, thus justifying the use of certain deterministic sequences instead of i.i.d. ones. In the remainder of the paper, we will refer to sequences with $\ell_{2}$-dispersion of order $O\left(n^{-1 / d}\right)$ as low-dispersion sequences. A natural question to ask is whether we can use a sequence that minimizes the $\ell_{2}$-dispersion. Unfortunately, such an optimal sequence is only known for $d=2$, in which case it is 
represented by the centers of the equilateral triangle tiling [Lavalle, 2006]. In this paper, we will focus on the Sukharev [Sukharev, 1971] and Halton sequences [Halton, 1960], except in two dimensions when we will consider the triangular lattice as well, though there are many other deterministic sequences with $\ell_{2}$-dispersion of order $O\left(n^{-1 / d}\right)$; see |Yershova and LaValle, 2004, Lindemann et al., 2005, Yershova et al., 2010] for other examples.

\section{Problem Statement}

The problem formulation follows that in [Janson et al. 2015] very closely. Let $\mathcal{X}=[0,1]^{d}$ be the configuration space, where $d \in \mathbb{N}$. Let $\mathcal{X}_{\text {obs }}$ be a closed set representing the obstacles, and let $\mathcal{X}_{\text {free }}=\operatorname{cl}\left(\mathcal{X} \backslash \mathcal{X}_{\text {obs }}\right)$ be the obstacle-free space, where $\mathrm{cl}(\cdot)$ denotes the closure of a set. The initial condition is $x_{\text {init }} \in \mathcal{X}_{\text {free }}$, and the goal region is $\mathcal{X}_{\text {goal }} \subset \mathcal{X}_{\text {free. }}$. A specific path planning problem is characterized by a triplet $\left(\mathcal{X}_{\text {free }}, x_{\text {init }}, \mathcal{X}_{\text {goal }}\right)$. A function $\sigma:[0,1] \rightarrow \mathbb{R}^{d}$ is a path if it is continuous and has bounded variation. If $\sigma(\tau) \in \mathcal{X}_{\text {free }}$ for all $\tau \in[0,1], \sigma$ is said to be collision-free. Finally, if $\sigma$ is collision-free, $\sigma(0)=x_{\text {init }}$, and $\sigma(1) \in \operatorname{cl}\left(\mathcal{X}_{\text {goal }}\right)$, then $\sigma$ is said to be a feasible path for the planning problem $\left(\mathcal{X}_{\text {free }}, x_{\text {init }}, \mathcal{X}_{\text {goal }}\right)$.

The goal region $\mathcal{X}_{\text {goal }}$ is said to be regular if there exists $\xi>0$ such that $\forall y \in \partial \mathcal{X}_{\text {goal }}$, there exists $z \in \mathcal{X}_{\text {goal }}$ with $B(z ; \xi) \subseteq \mathcal{X}_{\text {goal }}$ and $y \in \partial B(z ; \xi)$ (the notation $\partial \mathcal{X}$ denotes the boundary of set $\mathcal{X}$ ). Intuitively, a regular goal region is a smooth set with a boundary that has bounded curvature. Regularity is a technical condition we will use in our results, but is in fact quite weak, as nearly any goal region can be well-approximated by a regular goal region. Furthermore, we will say $\mathcal{X}_{\text {goal }}$ is $\xi$-regular if $\mathcal{X}_{\text {goal }}$ is regular for the parameter $\xi$. Denote the set of all paths by $\Sigma$. A cost function for the planning problem $\left(\mathcal{X}_{\text {free }}, x_{\text {init }}, \mathcal{X}_{\text {goal }}\right)$ is a function $c: \Sigma \rightarrow \mathbb{R}_{\geq 0}$; in this paper we will focus on the arc length function. The is then defined as follows:

Optimal path planning problem: Given a path planning problem $\left(\mathcal{X}_{\text {free }}, x_{\text {init }}, \mathcal{X}_{\text {goal }}\right)$ with an arc length cost function $c: \Sigma \rightarrow \mathbb{R}_{\geq 0}$, find a feasible path $\sigma^{*}$ such that $c\left(\sigma^{*}\right)=\min \{c(\sigma): \sigma$ is feasible $\}$. If no such path exists, report failure.

A path planning problem can be arbitrarily difficult if the solution traces through a narrow corridor, which motivates the standard notion of path clearance [Karaman and Frazzoli, 2011]. For a given $\delta>0$, define the $\delta$-interior of $\mathcal{X}_{\text {free }}$ as the set of all configurations that are at least a distance $\delta$ from $\mathcal{X}_{\text {obs }}$. Then a path is said to have strong $\delta$-clearance if it lies entirely inside the $\delta$-interior of $\mathcal{X}_{\text {free }}$. Further, a path planning problem with optimal path cost $c^{*}$ is called $\delta$-robustly feasible if there exists a strictly positive sequence $\delta_{n} \rightarrow 0$, and a sequence $\left\{\sigma_{n}\right\}_{i=1}^{n}$ of feasible paths such that $\lim _{n \rightarrow \infty} c\left(\sigma_{n}\right)=c^{*}$ and for all $n \in \mathbb{N}, \sigma_{n}$ has strong $\delta_{n}$-clearance, $\sigma_{n}(1) \in \partial \mathcal{X}_{\text {goal }}$, and $\sigma_{n}(\tau) \notin \mathcal{X}_{\text {goal }}$ for all $\tau \in(0,1)$.

Lastly, in this paper we will be considering a generic form of the PRM algorithm. That is, denote by gPRM (for generic PRM) the algorithm given by Algorithm 1 The function $\operatorname{SampleFree}(n)$ is a function that returns a set of $n \in \mathbb{N}$ points

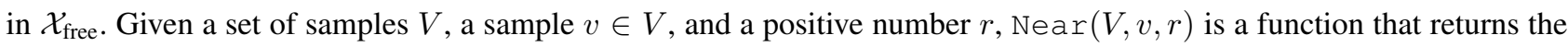
set of samples $\left\{u \in V:\|u-v\|_{2}<r\right\}$. Given two samples $u, v \in V$, Collisionfree $(u, v)$ denotes the boolean function which is true if and only if the line joining $u$ and $v$ does not intersect an obstacle. Given a graph $(V, E)$, where the node set $V$ contains $x_{\text {init }}$ and $E$ is the edge set, $\operatorname{ShortestPath}\left(x_{\text {init }}, V, E\right)$ is a function returning a shortest path from $x_{\text {init }}$ to $\mathcal{X}_{\text {goal }}$ in the graph $(V, E)$ (if one exists, otherwise it reports failure). Deliberately, we do not specify the definition of SampleFree and have left $r_{n}$ unspecified, thus allowing for any sequence of points-deterministic or random-to be used, with any connection radius. These "tuning" choices will be studied in Section 4 We want to clarify that we are in no way proposing a new algorithm, but just defining an umbrella term for the PRM class of algorithms which includes, for instance, sPRM and PRM* as defined in [Karaman and Frazzoli, 2011]. 


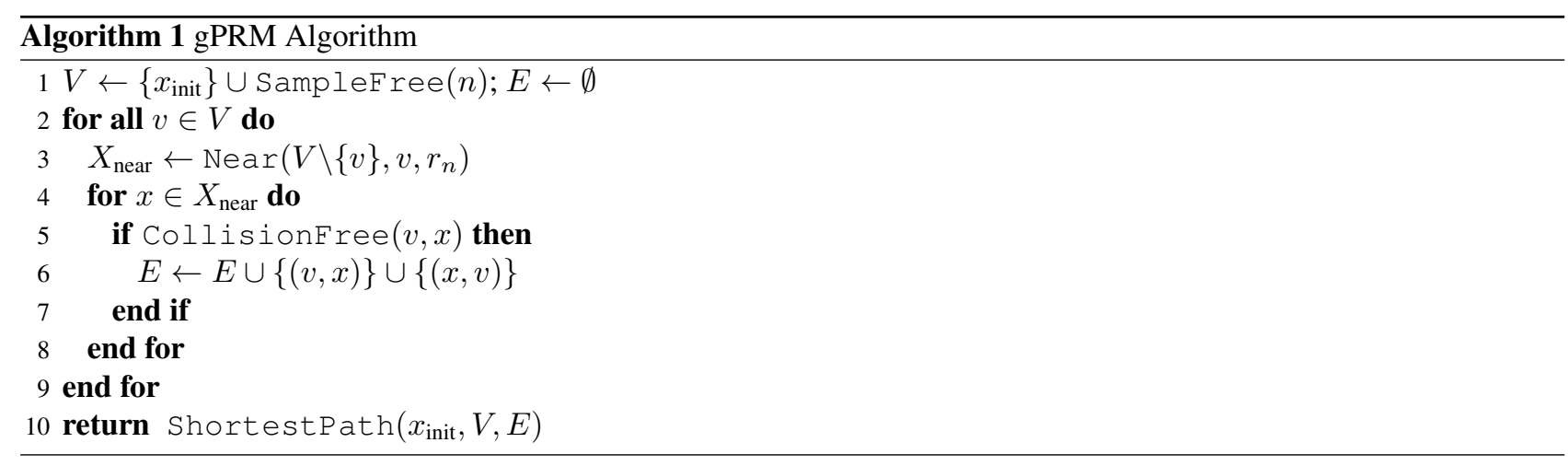

\section{Theoretical Results}

In this section we present our main theoretical results. We begin by proving that gPRM on low-dispersion sequences is asymptotically optimal, in the deterministic sense, for connection radius $r_{n} \in \omega\left(n^{-1 / d}\right)$. Previous work has required $r_{n}$ to be at least $\Omega\left((\log (n) / n)^{1 / d}\right)$ for asymptotic optimality.

Theorem 1 (Asymptotic optimality with deterministic sampling). Let $\left(\mathcal{X}_{\text {free }}, x_{\text {init }}, \mathcal{X}_{\text {goal }}\right)$ be a $\delta$-robustly feasible path planning problem in d dimensions, with $\delta>0$ and $\mathcal{X}_{\text {goal }} \xi$-regular. Let $c^{*}$ denote the arc length of an optimal path $\sigma^{*}$, and let $c_{n}$ denote the arc length of the path returned by $g P R M($ or $\infty$ if gPRM returns failure) with $n$ vertices whose $\ell_{2}$-dispersion is $D(V)$ using a radius $r_{n}$. Then if $D(V) \leq \gamma n^{-1 / d}$ for some $\gamma \in \mathbb{R}$ and

$$
n^{1 / d} r_{n} \rightarrow \infty
$$

then $\lim _{n \rightarrow \infty} c_{n}=c^{*}$.

Proof. Fix $\varepsilon>0$. By the $\delta$-robust feasibility of the problem, there exists a $\sigma_{\varepsilon}$ such that $c\left(\sigma_{\varepsilon}\right) \leq(1+\varepsilon / 3) c^{*}$ and $\sigma_{\varepsilon}$ has strong $\delta_{\varepsilon}$-clearance for some $\delta_{\varepsilon}>0$, see Figure 1(a) Let $R_{n}$ be a sequence such that $R_{n} \leq r_{n}, n^{1 / d} R_{n} \rightarrow \infty$, and

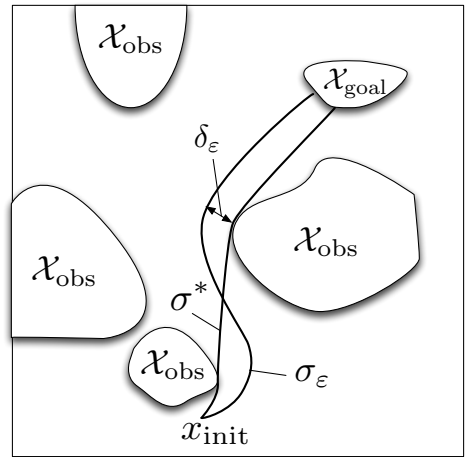

(a)

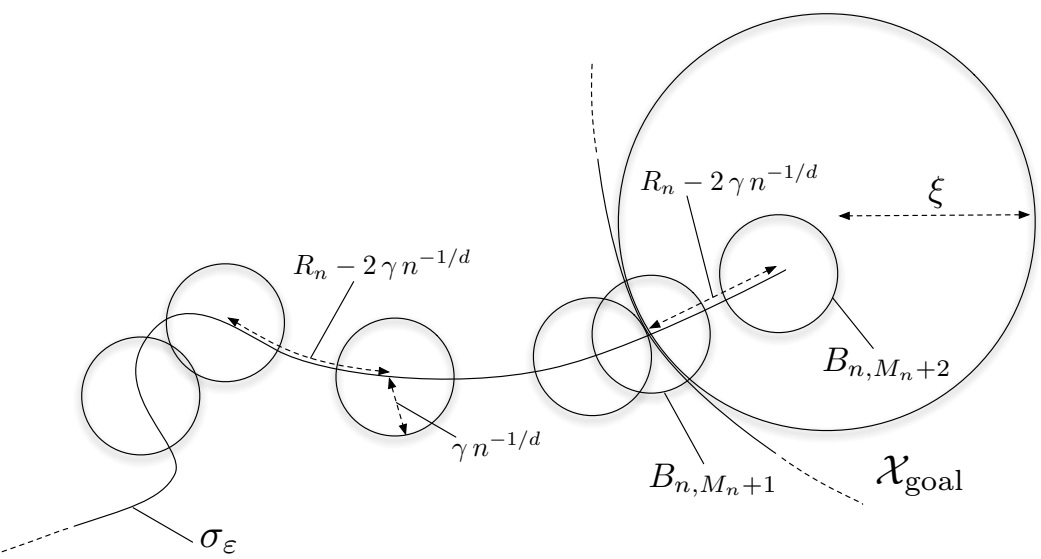

(b)

Fig. 1. Figure 1(a) Illustration in 2D of $\sigma_{\varepsilon}$ as the shortest strongly $\delta_{\varepsilon}$-robust feasible path, as compared to the optimal path $\sigma^{*}$, as used in the proof of Theorem 1 Figure $1(\mathrm{~b})$. Illustration in 2D of the construction of $B_{1}, \ldots, B_{M_{n}+2}$ in the proof of Theorem 1

$R_{n} \rightarrow 0$, guaranteeing that there exists a $n_{0} \in \mathbb{N}$ such that for all $n \geq n_{0}$,

$$
(4+6 / \varepsilon) \gamma n^{-1 / d} \leq R_{n} \leq \min \left\{\delta_{\varepsilon}, \xi, c^{*} \varepsilon / 6\right\} .
$$


For any $n \geq n_{0}$, construct the closed balls $B_{n, m}$ such that $B_{n, i}$ has radius $\gamma n^{-1 / d}$ and has center given by tracing a distance $\left(R_{n}-2 \gamma n^{-1 / d}\right) i$ from $x_{0}$ along $\sigma_{\varepsilon}$ (this distance is positive by equation (3) ) until $\left(R_{n}-2 \gamma n^{-1 / d}\right) i>c\left(\sigma_{\varepsilon}\right)$. This will generate $M_{n}=\left\lfloor c\left(\sigma_{\varepsilon}\right) /\left(R_{n}-2 \gamma n^{-1 / d}\right)\right\rfloor$ balls. Define $B_{n, M_{n}+1}$ to also have radius $\gamma n^{-1 / d}$ but center given by the point where $\sigma_{\varepsilon}$ meets $\mathcal{X}_{\text {goal }}$. Finally, define $B_{n, M_{n}+2}$ to have radius $\gamma n^{-1 / d}$ and center defined by extending the center of $B_{n, M_{n}+1}$ into $\mathcal{X}_{\text {goal }}$ by a distance $R_{n}-2 \gamma n^{-1 / d}$ in the direction perpendicular to $\partial \mathcal{X}_{\text {goal }}$. Note that by equation (3), $B_{n, M_{n}+2} \subset \mathcal{X}_{\text {goal }}$. See Figure $1(\mathrm{~b})$ for an illustration.

Since the dispersion matches the radii of all the $B_{n, m}$, each $B_{n, m}$ has at least one sampled point within it. Label these points $x_{1}, \ldots, x_{M_{n}+2}$, with the subscripts matching their respective balls of containment. For notational convenience, define $x_{0}:=x_{\text {init }}$. Note that by construction of the balls, for $i \in\left\{0, \ldots, M_{n}+1\right\}$, each pair of consecutively indexed points $\left(x_{i}, x_{i+1}\right)$ is separated by no more than $R_{n} \leq r_{n}$. Furthermore, since $R_{n} \leq \delta_{\varepsilon}$ by equation (3) above, there cannot be an obstacle between any such pair, and thus each pair constitutes an edge in the gPRM graph. Thus, we can upper-bound the cost $c_{n}$ of the gPRM solution by the sum of the lengths of the edges $\left(x_{0}, x_{1}\right), \ldots,\left(x_{M_{n}+1}, x_{M_{n}+2}\right)$ :

$$
\begin{aligned}
c_{n} & \leq \sum_{i=0}^{M_{n}+1}\left\|x_{i+1}-x_{i}\right\| \leq\left(M_{n}+2\right) R_{n} \leq \frac{c\left(\sigma_{\varepsilon}\right)}{R_{n}-2 \gamma n^{-1 / d}} R_{n}+2 R_{n} \\
& \leq c\left(\sigma_{\varepsilon}\right)+\frac{2 \gamma n^{-1 / d}}{R_{n}-2 \gamma n^{-1 / d}} c\left(\sigma_{\varepsilon}\right)+2 R_{n}=c\left(\sigma_{\varepsilon}\right)+\frac{1}{\frac{R_{n}}{2 \gamma n^{-1 / d}}-1} c\left(\sigma_{\varepsilon}\right)+2 R_{n} \\
& \leq\left(1+\frac{\varepsilon}{3}\right) c^{*}+\frac{1}{\frac{3}{\varepsilon}+1}\left(1+\frac{\varepsilon}{3}\right) c^{*}+\frac{\varepsilon}{3} c^{*}=(1+\varepsilon) c^{*} .
\end{aligned}
$$

The second inequality follows from the fact that the distance between $x_{i}$ and $x_{i+1}$ is upper-bounded by the distance between the centers of $B_{n, i}$ and $B_{n, i+1}$ (which is at most $R_{n}-2 \gamma n^{-1 / d}$ ) plus the sum of their radii (which is $2 \gamma n^{-1 / d}$ ). The last inequality follows from the facts that $c\left(\sigma_{\varepsilon}\right) \leq(1+\varepsilon / 3) c^{*}$ and equation (3).

Note that if gPRM using $r_{n}>2 D(V)$ reports failure, then there are two possibilities: (i) a solution does not exist, or (ii) all solution paths go through corridors whose widths are smaller than $2 D(V)$. Such a result can be quite useful in practice, as solutions going through narrow corridors could be undesirable anyways (see [LaValle et al., 2004, Section 5] for the same conclusion).

Next, we relate the solution cost returned by gPRM to the best cost of a path with strong $\delta$-clearance in terms of the $\ell_{2}$-dispersion of the samples used. This is a generalization of previous convergence rates, e.g. [Janson et al., 2015], which only apply to obstacle-free spaces. Previous work also defined convergence rate as, for a fixed level of suboptimality $\varepsilon$, the rate (in $n$ ) that the probability of returning a greater-than- $\varepsilon$-suboptimal solution goes to zero. In contrast, we compute the rate (in $n$ ) that solution suboptimality approaches zero. Lastly, previous work focused on asymptotic rates in big- $O$ notation, while here we provide exact upper-bounds for finite samples.

Theorem 2 (Convergence rate in terms of dispersion). Consider the simplified problem of finding the shortest feasible path between two points $x_{0}$ and $x_{f}$ in $\mathcal{X}_{\text {free }}$, assuming that both the initial point and final point have already been sampled. Define

$$
\delta_{\max }=\sup \{\delta>0: \exists \text { a feasible } \sigma \in \Sigma \text { with strong } \delta \text {-clearance }\}
$$

and assume $\delta_{\max }>0$. For all $\delta<\delta_{\max }$, let $c^{(\delta)}$ be the cost of the shortest path with strong $\delta$-clearance. Let $c_{n}$ be the length of the path returned by running gPRM on $n$ points whose $\ell_{2}$-dispersion is $D_{n}$ and using a connection radius $r_{n}$. Then for all $n$ such that $r_{n}>2 D_{n}$ and $r_{n}<\delta$,

$$
c_{n} \leq\left(1+\frac{2 D_{n}}{r_{n}-2 D_{n}}\right) c^{(\delta)} .
$$


Proof. Let $\sigma_{\delta}$ be a feasible path of length $c^{(\delta)}$ with strong $\delta$-clearance. Construct the balls $B_{1}, \ldots, B_{M_{n}}$ with centers along $\sigma_{\delta}$ as in the proof of Theorem 1 (note we are not constructing $B_{M_{n}+1}$ or $B_{M_{n}+2}$ ), except with radii $D_{n}$ and centers separated by a segment of $\sigma_{\delta}$ of arc-length $r_{n}-2 D_{n}$. Note that $M_{n}=\left\lfloor c^{(\delta)} /\left(r_{n}-2 D_{n}\right)\right\rfloor$. Then by definition each $B_{i}$ contains at least one point $x_{i}$. Furthermore, each $x_{i}$ is connected to $x_{i-1}$ in the gPRM graph (because $x_{i}$ is contained in the ball of radius $r_{n}-D_{n}$ centered at $x_{i-1}$, and that ball is collision-free), and $x_{f}$ is connected to $x_{M_{n}}$ as well. Thus $c_{n}$ is upper-bounded by the path tracing through $x_{0}, x_{1}, \ldots, x_{M_{n}}, x_{f}$ :

$$
\begin{aligned}
c_{n} & \leq\left\|x_{1}-x_{0}\right\|+\sum_{i=2}^{M_{n}}\left\|x_{i}-x_{i-1}\right\|+\left\|x_{f}-x_{M_{n}}\right\| \leq r_{n}-D_{n}+\sum_{i=2}^{M_{n}} r_{n}+\left\|x_{f}-x_{M_{n}}\right\| \\
& \leq\left(M_{n} r_{n}-D_{n}\right)+\left(\left(\frac{c^{(\delta)}}{r_{n}-2 D_{n}}-\left\lfloor\frac{c^{(\delta)}}{r_{n}-2 D_{n}}\right\rfloor\right)\left(r_{n}-2 D_{n}\right)+D_{n}\right) \\
& =c^{(\delta)}+2 D_{n} M_{n} \leq\left(1+\frac{2 D_{n}}{r_{n}-2 D_{n}}\right) c^{(\delta)},
\end{aligned}
$$

where the second and third inequalities follow by considering the farthest possible distance between neighboring points, given their inclusion in their respective balls.

Remark 1 (Convergence rate in obstacle-free environments). Note that when there are no obstacles, $\delta_{\max }=\infty$ and $c^{(\delta)}=\left\|x_{f}-x_{0}\right\|$ for all $\delta>0$. Therefore, an immediate corollary of Theorem 2 is that the convergence rate in free space of gPRM to the optimal solution is upper-bounded by $2 D_{n} /\left(r_{n}-2 D_{n}\right)$ for $r_{n}>2 D_{n}$.

Remark 2 (Practical use of convergence rate). Theorem 2 provides a convergence rate result to a shortest path with strong $\delta$-clearance. This result is useful for two main reasons. First, in practice, the objective of path planning is often to find a high-quality path with some "buffer distance" from the obstacles, which is precisely captured by the notion of $\delta$-clearance. Second, the convergence rate in equation (4) could be used to certify the performance of gPRM (and related batch planners) by placing some assumptions on the obstacle set (e.g., minimum separation distance among obstacles and/or curvature of their boundaries). For instance, consider a deterministic low-dispersion sequence with dispersion upper bounded by $\gamma n^{-1 / d}$, and assume one has time to plan on $\bar{n}$ samples. Choose $r_{\bar{n}}=2 \psi \gamma \bar{n}^{-1 / d}$ for some $\psi>1$ and choose $\delta_{0}>r_{\bar{n}}$ (one can readily verify that such a choice of $r_{\bar{n}}$ satisfies the assumptions of Theorem 2). Then one can state the deterministic guarantee "For all planning problems where there exists a feasible $\delta_{0}$-clear path (see Figure 2), the cost of the path returned is within a factor $1 /(\psi-1)$ of the cost of the best $\delta_{0}$-clear path." For given values of $\psi$ and $\delta_{0}$, one can therefore "certify" the performance of the planner for a desired target performance. An interesting avenue for future research is to use information about the curvature of the obstacles to quantify the difference between $c^{(\delta)}$ and the true optimal cost $c^{*}$.

Both the asymptotic optimality and convergence rate results can be extended to other batch planners such as Lazy-PRM or FMT* ${ }^{*}$ as discussed in Section 5.1 .

Lastly, we show that using a low- $\ell_{2}$-dispersion lattice sample set, an asymptotically-optimal (AO) version of gPRM can be run that has lower-order computational complexity than any existing AO algorithm, namely $\omega(n)$ instead of $O(n \log (n))$.

Theorem 3 (Computational complexity with deterministic sampling). gPRM run on $n$ samples arranged in a d-dimensional cubic lattice with connection radius $r_{n}$ satisfying equation 2 has computational complexity

$$
O\left(n^{2} r_{n}^{d}\right)
$$

Furthermore, as long as $n r_{n}^{d} \nrightarrow 0$, the computational complexity is also $O\left(n^{2} r_{n}^{d}\right)$.

Proof. The algorithm gPRM has three steps: (1) For each sampled point $x$, it needs to compute which other sampled points are within a distance $r_{n}$ of $x$. (2) For each pair of sampled points within $r_{n}$ of one another, their connecting edge needs 


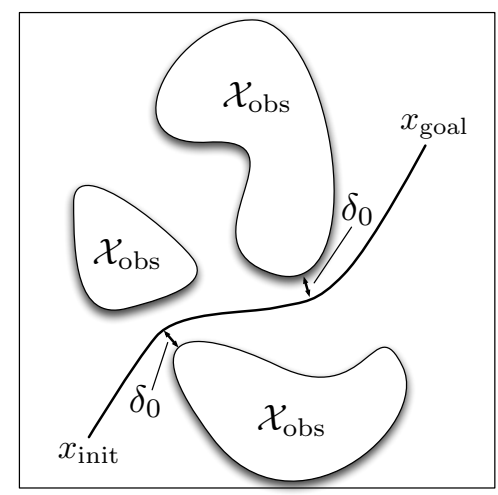

Fig. 2. An example of a planning problem with a feasible $\delta_{0}$-clear path. For a given clearance parameter $\delta_{0}$ and suboptimality factor $\psi$, one can readily determine the number of samples (and, hence, computation time) that is deterministically guaranteed to return a path whose cost is within $1 /(\psi-1)$ of the cost of the best $\delta_{0}$-clear path, for all planning problems where a feasible $\delta_{0}$-clear path exists.

to be checked for collision, and if collision-free, its edge-length needs to be computed. (3) The shortest path through the graph produced in steps (1) and (2) from the initial point to the goal region needs to be computed.

The lattice structure makes it trivially easy to bound a point's $r_{n}$-neighborhood by a bounding hypercube with sidelength $2 r_{n}$, ensuring only $O\left(n r_{n}^{d}\right)$ nearby points need to be checked for each of the $n$ samples, so this step takes $O\left(n^{2} r_{n}^{d}\right)$ time.

In step (2), one collision-check and at most one cost computation needs to be performed for each pair of points found in step (1) to be within $r_{n}$ of one another. The number of such pairs can be bounded above by the number of sampled points times the size of each one's neighborhood, leading to a bound of the form $O\left(n \cdot n r_{n}^{d}\right)$. Thus step (2) takes $O\left(n^{2} r_{n}^{d}\right)$ time.

After steps (1) and (2), a weighted (weights correspond to edge lengths) graph has been constructed on $n$ vertices with a number of edges asymptotically upper-bounded by $n^{2} r_{n}^{d}$. One more property of this graph, because it is on the cubic lattice, is that the number of distinct edge lengths is asymptotically upper-bounded by $n r_{n}^{d}$. An implementation of Dijkstra's algorithm for the single source shortest path problem is presented in [Orlin et al. 2010] with running time linear in both the number of edges and the number of vertices times the number of distinct edge lengths. Since both are $O\left(n^{2} r_{n}^{d}\right)$, that is the time complexity of step (3).

The space complexity is proportional to the number of edges plus the number of vertices, which are $O\left(n^{2} r_{n}^{d}\right)$ and $O(n)$, respectively. By assumption that $n r_{n} \nrightarrow 0, O\left(n^{2} r_{n}^{d}\right)$ will be the (possibly co-) dominant term.

Since Theorem 1 allows $r_{n} \in \omega\left(n^{-1 / d}\right)$ while maintaining AO, Theorem 3 implies that cubic-lattice sampling allows for an $\mathrm{AO}$ algorithm with computational and space complexity $\omega(n)$. All other $\mathrm{AO}$ algorithms in the literature have computational and space complexity at least $O(n \log (n))$. While the use of an $r_{n} \in \omega\left(n^{-1 / d}\right)$ makes the graph construction phase (steps (1) and (2)) $\omega(n)$, step (3) would in general take longer, as shortest-path algorithms on a general graph with $n$ vertices requires $\Omega(n \log (n))$. Thus the lattice structure must be leveraged to improve the complexity of step (3) —we discuss the limitations implied by this in the next section.

\section{Extensions}

In this section we discuss several extensions to the results presented in Section 4 In particular, we discuss extensions to alternative implementations and other types of batch-processing algorithms (Sections 5.1 and 5.2), to non-uniform sampling sequences (Section 5.3), and to kinodynamic motion planning (Section 5.4. 


\subsection{Convergence Results for Other Batch-Processing Algorithms}

The theoretical convergence results of the previous section (Theorems 1 and 2) extend to alternative implementations of gPRM and other batch-processing algorithms. We first discuss a variant of gPRM which makes connections based on $k$-nearest-neighbors instead of a fixed connection radius. This variant, referred to as $k$-nearest gPRM, has the advantage of being more adaptive to different obstacle spaces than its connection-radius counterpart (we refer the reader to [Janson et al.

2015. Section 5.3] for a more detailed discussion about the relative benefits of a $k$-nearest-neighbors implementation). Assuming that $k$-nearest gPRM is implemented so that the number of neighbors $k_{n}$ (a function of $n$, as $r_{n}$ ) is taken for each sample to be roughly connected to its $r_{n}$-neighborhood if there were no obstacles, then deterministic asymptotic optimality and rate convergence guarantees can be readily derived. Specifically, assuming $k_{n}=(1+\epsilon) n \zeta_{d} r_{n}^{d}$, where $\epsilon>0$ and $\zeta_{d}$ is the volume of the unit ball in $d$ dimensions, then a graph constructed in $k$-nearest gPRM with $k_{n}$ neighbors will asymptotically contain all the edges (and more) of the graph constructed in gPRM with radius $r_{n}$, and thus will return a path at least as low-cost.

A second example is Lazy-PRM [Bohlin and Kavraki] 2000], where the extension of the theoretical convergence results is straightforward, as the path returned by Lazy-PRM is identical to that returned by gPRM (using the same radius).

A third example is FMT* [Janson et al. 2015]. The only difference in the proof for FMT* from that for gPRM is that $c_{n}$ cannot naïvely be upper-bounded by the length of a path tracing through a sequence of points in the gPRM graph, as FMT* may not use some edges. However, as shown in the proof of Lemma 4.2 (pages 912-913) in [Janson et al., 2015. Appendix A], FMT*'s $c_{n}$ can be bounded by the length of a path tracing through a sequence of points in the gPRM graph if those points are contained in a suitable sequence of balls. In particular, the sequence of balls needs to be sufficiently far from obstacles and adjacent balls need to be sufficiently close together. It is easy to check that the sequences $\left\{B_{i}\right\}$ used in the proofs of the previous section satisfy these conditions.

\subsection{Complexity Results for Different Sampling Schemes and Other Batch-Processing Algorithms}

The complexity result in Theorem 3 strictly speaking, only applies to gPRM as defined in Algorithm 1 run on $n$ samples arranged in a $d$-dimensional cubic lattice. Using other low-dispersion but non-lattice sampling schemes, such as the Halton/Hammersley sequence, would not give $O\left(n r_{n}^{d}\right)$ distinct edge lengths, which precludes the use of the improved implementation of Dijkstra's algorithm [Orlin et al. [2010]. A $k$-nearest-neighbor implementation (where $k_{n}$ is selected as specified in Section 5.1 in order to ensure convergence), even on a lattice, would also no longer in general have $O\left(n r_{n}^{d}\right)$ distinct edge lengths. Lastly, other batch-processing algorithms, such as FMT*, do not separate graph construction and shortest-path computation, again precluding, at least naïvely, the use of the improved implementation of Dijkstra's algorithm.

In all the above cases, all the same computational advantages stated in the proof of Theorem 3 would hold, except the advantage from the sped-up shortest-path algorithm in step (3). In practice the shortest-path algorithm is typically a trivial fraction of path planning runtime, as it requires none of the much-more-complex edge-cost and collision-free computations. In other words, the constant hidden in the big-O notation for the shortest-path algorithm is drastically smaller than that in the other steps. Thus, a practical approximation of the runtime could ignore the shortest-path component, in which case the result of Theorem 3 can be extended to all of the aforementioned examples.

\subsection{Non-uniform Sampling}

A popular method for incorporating prior knowledge about a problem is to draw samples in a way that is not uniform throughout the configuration space. Increased sampling density in areas that are especially hard to traverse gives a motion planning algorithm help in that area. Thus if these areas can be identified a priori or even adaptively (see, for example, [Boor et al. 1999|), observed convergence can be substantially sped up. Sampling non-uniformly in the i.i.d. setting is often fairly straightforward, for instance by simply adding sample-rejection rules to the uniform strategy [Hsu et al. 2006]. As long as 
there remains a baseline density of samples everywhere in the space, similar worst-case (i.e., even if the non-uniformity of sampling was poorly chosen for the problem at hand) guarantees to the uniform case can still be made (see, for example, [Janson et al. 2015]). The notion of density is less clear in the deterministic case, but we can fall back on the notion of $\ell_{2}$-dispersion instead. Indeed, the theoretical convergence results in this paper are not specific to any deterministic sampling strategy, but simply make a requirement on the $\ell_{2}$-dispersion. Thus, many non-i.i.d., non-uniform sampling strategies fit into the analysis given here. Consider, for instance, using a sequence with $\ell_{2}$-dispersion upper-bounded by $\gamma n^{-1 / d}$ to sample $n / 2$ points, and then sampling $n / 2$ more points in any way (e.g., biased towards sampling near obstacles). The resulting set of $n$ points will have $\ell_{2}$-dispersion upper-bounded by $2^{1 / d} \gamma n^{-1 / d}$ and thus still be a low- $\ell_{2}$-dispersion sequence. There is a rich literature in different methods for non-uniform sampling, and an interesting future direction will be to investigate how best to adapt such methods to the deterministic context.

\subsection{Deterministic Kinodynamic Planning}

Another important extension is to motion planning with differential constraints. In particular, we consider here the extension to systems with linear affine dynamics of the form: $\dot{\mathbf{x}}(t)=A \mathbf{x}(t)+B \mathbf{u}(t)+\mathbf{c}$, where $A, B$, and $\mathbf{c}$ are constants. The extension of the $\ell_{2}$-dispersion-based analysis of this paper to that case poses some challenges. The key roadblock is that the $\ell_{2}$-dispersion is no longer a particularly accurate measure of how suitable a set of points is to track an optimal differentiallyconstrained path. Essentially, Euclidean balls must be replaced by "perturbation" balls [Schmerling et al. 2015b], which are high-dimensional ellipses. To be clear, by a high-dimensional ellipse we mean a volume defined by

$$
\left\{x: x^{T} Q x<r\right\}
$$

for some positive-definite matrix $Q$ and scalar $r$. Although such ellipses may be inner-bounded by a Euclidean ball, this (poor) approximation adds an exponential factor of the controllability index of the pair $(A, B)$ [Schmerling et al. 2015b] to the analysis. (Assuming the pair $(A, B)$ is controllable, the controllability indices $\nu_{i}$ give a fundamental notion of how difficult a linear system is to control in the various directions, see [Kailath, 1980, p. 431] or [Chen, 1995, pp. 150] for a detailed treatment. The number of controllability indices is equal to the number of control inputs, that is to the number of columns of $B$. The maximum, that is $\nu=\max \nu_{i}$, is referred to as the controllability index of the pair $(A, B)$.) The following theorem (whose proof is largely based on the analysis framework devised in [Schmerling et al. 2015b]) summarizes the optimality result. Here gDPRM is just Algorithm 1 except that Near uses the cost in [Schmerling et al., 2015b, equation (2)] instead of arc-length.

Theorem 4 (Asymptotic optimality with deterministic sampling for systems with linear affine dynamics). Under the assumptions of [Schmerling et al. 2015b. Theorem VI.1], gDPRM with deterministic low-dispersion sampling is asymptotically-optimal for

$$
r_{n}=C_{1} n^{-1 /(\nu d)}
$$

for some constant $C_{1}$, while gDPRM with i.i.d. uniform sampling is asymptotically optimal for

$$
r_{n}=C_{2}\left(\frac{\log (n)}{n}\right)^{1 / \tilde{D}}
$$

for some constant $C_{2}$, where $\tilde{D}=\left(d+\sum \nu_{i}^{2}\right) / 2$, the $\nu_{i}$ are the controllability indices of the pair $(A, B)$, and $\nu=\max \nu_{i}$.

Proof sketch. For the sake of brevity, we only describe here the changes needed to the theory in [Schmerling et al. 2015b], as the results have much in common. The proof of (7) is nearly identical to that of [Schmerling et al. 2015b Theorem VI.1] except with a low-dispersion analogue of [Schmerling et al. 2015b, Theorem IV.6] which uses the same $r_{n}$ rate as 
in (7). To see this analogue result, note that [Schmerling et al. 2015b, Lemma IV.5] holds deterministically under lowdispersion sampling with the log term removed from the condition on the volume $\mu\left[T_{k}\right]$. Then the proof of the analogue of [Schmerling et al. 2015b. Theorem IV.6] only requires construction of the sets $S_{k}$ (no $T_{k}$ ), again without the log term. From the deterministic version of [Schmerling et al. 2015b, Lemma IV.5], we have that for the scaling of $r_{n}$ as in (7), every set $S_{k}$ will contain a sample, and equation (7) follows.

Finally, equation (8) is a direct corollary of [Schmerling et al., 2015b, Theorem VI.1].

If $\nu=1$ (i.e., all directions are "equally difficult" to control), deterministic sampling and our analysis show all the same benefits as in the case of the path planning (non-kinodynamic) problem by getting rid of the $\log (n)$ term required by i.i.d. sampling without changing the exponent (as in this case, $\nu d=d$ and $\tilde{D}=d$ ). Note that a special case where $\nu=1$ is represented by the single-integrator model $\dot{\mathbf{x}}(t)=\mathbf{u}(t)$, which effectively reduces the kinodynamic planning problem to the path planning problem stated in Section 3 -in this sense, Theorem 7 recovers Theorem 1 when $\dot{\mathbf{x}}(t)=\mathbf{u}(t)$. However, in general, the exponent for the case of deterministic low-dispersion sampling (i.e., the exponent in equation (7)) may be worse. For instance, for the double-integrator model in three dimensions, namely $\ddot{\mathbf{x}}(t)=\mathbf{u}(t)$ and $d=6$, the three controllability indices are $\nu_{1}=\nu_{2}=\nu_{3}=2$. As a consequence, one obtains $\nu d=12$ and $\tilde{D}=9$, and the radius in equation (7) (i.e., for the deterministic case) is larger that the radius for equation (8) (i.e., for the case with i.i.d. uniform sampling).

This is not to say that deterministic sampling is necessarly inappropriate or not advantageous for differentiallyconstrained problems, but just that the analysis used here is inadequate (most critically, we crudely inner-bound ellipses via Euclidean balls). Our analysis does, however, suggest possible ways forward. One could consider a measure of dispersion which applies more specifically to ellipses, and possibly tailor a deterministic sequence to be low-dispersion in this sense. To our knowledge, no assessment of sample sequences in terms of this type of dispersion has been performed previously, and this represents a theoretically and practically important direction for future research (together with studying tailored notions of sampling sequences and dispersion for other classes of dynamical systems, e.g., driftless systems).

We will further study kinodynamic motion planning via deterministic sampling sequences through numerical experiments in Section6

\section{Numerical Experiments}

In this section we numerically investigate the benefits of planning with deterministic low-dispersion sampling instead of i.i.d. sampling. Section 6.1 overviews the simulation environment used for this investigation. Section 6.2 details the deterministic low-dispersion sequences used, namely, lattices and the Halton sequence. Several simulations are then introduced and results compared to i.i.d. sampling in Sections 6.3 and 6.4 Finally, we briefly discuss non-i.i.d., random, low-dispersion sampling schemes in Section 6.5.

\subsection{Simulation Environment}

Simulations were written in C++, MATLAB, and Julia [Bezanson et al. 2012], and run using a Unix operating system with a $2.3 \mathrm{GHz}$ processor and $8 \mathrm{~GB}$ of RAM. The $\mathrm{C}++$ simulations were run through the Open Motion Planning Library (OMPL) [Şucan et al., 2012]. The planning problems simulated in OMPL were rigid body problems, the simulations in MATLAB involved point robots and kinematic chains, and those in Julia incorporated kinodynamic constraints. For each planning problem, the entire implementation of gPRM was held fixed (including the sequence of $r_{n}$ ) except for the sampling strategy. Specifically, for all simulations (except the chain and kinodynamic simulations), we use as connection radius $r_{n}=\gamma_{\mathrm{PRM}}(\log (n) / n)^{1 / d}$, where $\gamma_{\mathrm{PRM}}=2.2(1+1 / d)^{1 / d}\left(1 / \zeta_{d}\right)^{1 / d}$ and $\zeta_{d}$ is again the volume of the unit ball in $d$-dimensional Euclidean space. This choice ensures asymptotic optimality both for i.i.d. sample sequences [Karaman 
and Frazzoli, 2011, Janson et al., 2015] and deterministic low-dispersion sequences (Theorem 11). Because this is an exact "apples-to-apples" comparison, we do not present runtime results but only results in terms of sample count, which have the advantage that they do not depend on the specific hardware or software we use. (Note that drawing samples represents a trivial fraction of the total algorithm runtime. Furthermore, as mentioned in the introduction, deterministic sampling even allows for possible speedups in computation.) The code for these results can be found at https://github.com/stanfordasl

\subsection{Sampling Sequences}

We consider two deterministic low-dispersion sequences, namely the Halton sequence [Halton, 1960] and lattices. Halton sampling is based on a generalization of the van der Corput sequence and uses prime numbers as its base to form a deterministic low-dispersion sequence of points [Halton, 1960, LaValle et al.,2004]. Lattices in this work were implemented as a triangular lattice in two dimensions and a Sukharev grid in higher dimensions [Sukharev, 1971].

Along with the benefits described throughout the paper, lattices also present some challenges [LaValle et al., 2004]. First, for basic cubic lattices with the same number of lattice points $k$ per side, the total number of samples $n$ is constrained to $k^{d}$, which limits the potential number of samples. For example, in 10 dimensions, the first four available sample counts are $1,1024,59,049$, and 1,048,576. There are some strategies to allow for incremental sampling |Yershova and LaValle, 2004, Lindemann et al. 2005, Yershova et al., 2010], but in this paper we overcome this difficulty by simply "weighting" dimensions. Explicitly, we allow each side to have a different number of lattice points. Independently incrementing each side's number of points by 1 increases the allowed resolution of $n$ by a factor of $d$, as it allows all numbers of the form $n=(k-1)^{m}(k)^{d-m}$.

Second, lattices are sensitive to axis-alignment effects, whereby an axis-aligned obstacle can invalidate an entire axisaligned row of samples. A simple solution to this problem is to rotate the entire lattice by a fixed amount (note this changes nothing about the $\ell_{2}$-dispersion or nearest-neighbor sets). We chose to rotate each dimension by $10 \pi$ degrees as an arbitrary angle far from zero (in practice, problems often show a "preferential" direction, e.g., vertical, so there may be an a priori natural choice of rotation angle).

Finally, we include examples in SE(2) and SE(3) because they are standard, even though the cost function is no longer arc-length in the configuration space, but closer to arc-length in the translational dimensions. As this is technically outside the theory in this paper, we must consider an appropriate analogue of deterministic low-dispersion sampling. In the naïve Sukharev lattice, each translational lattice point represents many rotational orientations. For instance, in SE(3), this means there are only $k^{3}$ translational points when $n=k^{6}$, providing a poor extension of the theory in this paper to the rigid body planning problem. A better approximate extension is to "spread" the points, which entails de-collapsing all the rotational orientations at each translational lattice point by spreading them deterministically in the translational dimensions around the original point. An example of this process with one rotational and two spatial dimensions is shown in Figure 3 . A similar phenomenon occurs with kinodynamic sampling, in which case it is beneficial to spread velocity samples. For the double-integrator model, as shown in Figure 4, this process entails offsetting velocity samples from their original positions by an amount proportional to their velocities. To then reduce unfavorable structure between neighboring lattice points and increase variation in connection types, alternating samples were rotated 180 degrees for the final implementation in Figure 4(c),

\subsection{Simulation Test Cases}

Within the MATLAB environment, results were simulated for a point robot within an Euclidean unit hypercube with a variety of geometric obstacles over a range of dimensions. First, rectangular obstacles in 2D and 3D were generated with a fixed configuration that allowed for several homotopy classes of solutions. A 2D maze with rectangular obstacles was also created (Figure 5(a)]. These sets of rectangular obstacles are of particular interest as they represent a possible "worst-case" 

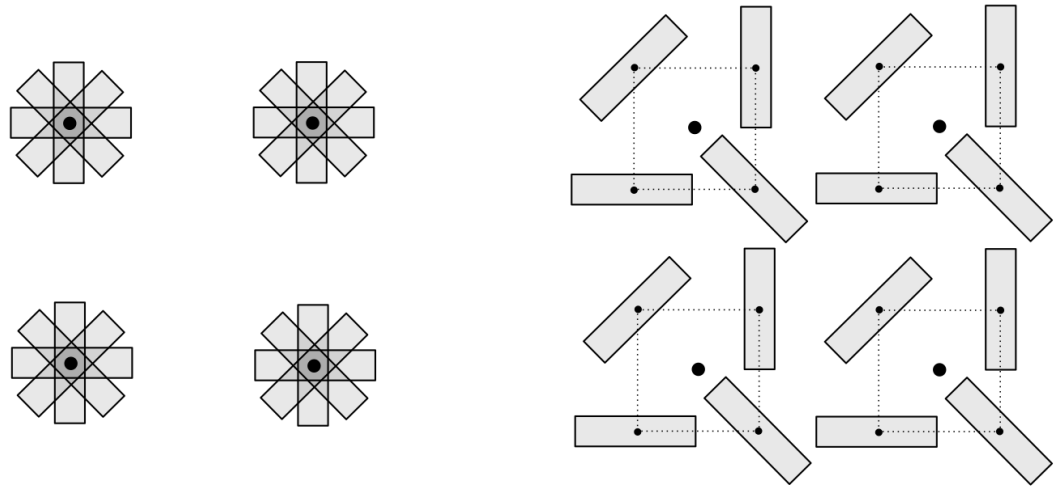

(a)

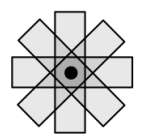

(b)

Fig. 3. Figure 3(a) Rectangular rigid body lattice samples with one rotational and two spatial degrees of freedom. Figure 3(b) A "spread" lattice formed by spreading the different rigid body orientations spatially around the original point results in a better spatial coverage of the configuration space.

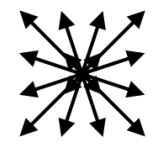

(a)

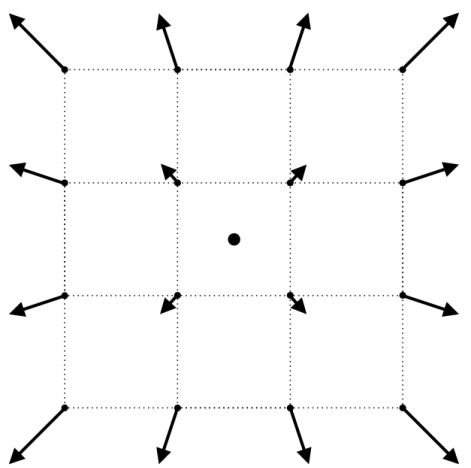

(b)

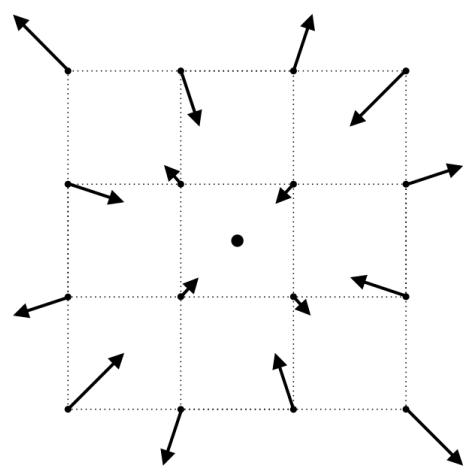

(c)

Fig. 4. Figure 4(a) A lattice for the double-integrator model with two spatial and two velocity degrees of freedom. Figure 4(b) A "spread" lattice formed by offsetting the velocity samples from their original positions (by an amount proportional to their velocities) to obtain better spatial coverage. Figure 4(c). The same lattice with alternating samples rotated 180 degrees to increase variation in types of connections and reduce unfavorable structure (this implementation was used for our results).

for lattice-based sampling because of the potential for axis alignment between samples and obstacles. The results, shown for the 2D maze in Figure 5 and for all experiments in Table 1, show that Halton and lattice sampling outperform i.i.d. sampling in both success rate and solution cost.

To illustrate planning with rectangular obstacles in higher dimensional space, we constructed a recursive maze obstacle environment. Each instance of the maze consists of two copies of the previous dimension's maze, separated by an obstacle with an opening through the new dimension, as detailed in [Janson et al., 2015]. Figure 6(a)] shows the maze in 2D and Figure 6(b) shows the maze in 3D with the two copies of the 2D maze in black and the opening in red. Halton and lattice sampling conferred similar benefits in the recursive mazes in $2 \mathrm{D}, 3 \mathrm{D}, 4 \mathrm{D}, 5 \mathrm{D}, 6 \mathrm{D}$, and $8 \mathrm{D}$ as they did in other simulations (see Table 1).

Along with the rectangular obstacles, hyperspherical obstacles within a Euclidean unit hypercube were generated to compare performance on a smooth obstacle set with no possibility of axis alignment between samples and obstacles. The setups for 2D and 3D (Figures 6(c) and 6(d) were fixed, while in 4D, obstacles were randomly generated to match a specified spatial coverage. Again, Halton and lattice sampling consistently outperformed random sampling, as shown in Table 1 . 


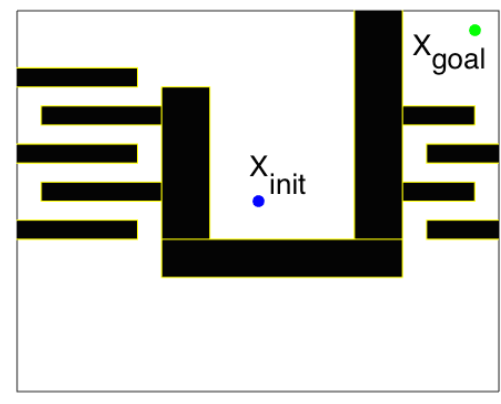

(a)

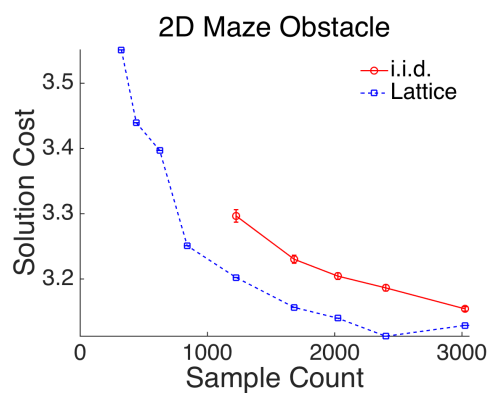

(b)

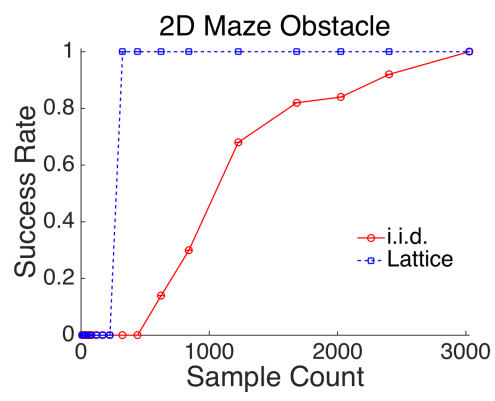

(c)

Fig. 5. Figure 5(a) The planning setup for a point robot with rectangular obstacles in a 2D maze. Figures 5(b) and 5(c) The results for solution cost and success rate versus sample count (averaged over 50 runs). For clarity we only report data for i.i.d. sampling and lattice sequences, results including Halton sampling are reported in Table 1 Only points with greater than 50\% success are shown in Figure $5(\mathrm{~b})$

As an additional high-dimensional example, an 8D kinematic chain planning problem with rotational joints, eight links, and a fixed base was created (Figure 6(e)]. The solution required the chain to be extracted from one opening and inserted into the other, as inspired by [Lindemann et al.,2005]. The chain cost function was set as the sum of the absolute values of the angle differences over all links and the connection radius was thus scaled by $\sqrt{d} \pi$. With this high dimension and new cost function, the Halton and lattice still perform as well as or better than i.i.d. sampling (see Table 1 ).

Within the OMPL environment, rigid body planning problems from the OMPL test banks were posed for SE(2) and $\mathrm{SE}(3)$ configuration spaces. In the SE(2) case, one rotational and two translational degrees of freedom are available, resulting in a three dimensional space, shown in Figure 6(f). The SE(3) problem consists of an "L-shaped" robot moving with three translational and three rotational degrees of freedom, resulting in six total problem dimensions, shown in Figure 6(g) As already mentioned, the rigid body planning problems are not strictly covered by the theory in this paper, and thus the $\mathrm{SE}(2)$ and SE(3) lattices use the spreading method described in Section 6.2. The results, summarized in Table 1 , show that Halton and lattice sampling generally outperform i.i.d. random sampling.

Lastly, planning problems with kinodynamic constraints were simulated within the Julia environment using the gDPRM algorithm defined in Section 5.4, with reference to [Schmerling et al. 2015b]. The connection radius was computed using equation (8) in Theorem 4. First, a double-integrator model with two spatial dimensions and two velocity dimensions was posed to simulate a system with drift (Figure 6(h) . To obtain better spatial coverage, the lattice was spread as described in Section 6.2. Second, results were simulated for the Reeds-Shepp car system [Reeds and Shepp, 1990], a regular driftless control-affine system with one rotational and two translational degrees of freedom (Figure 6(h)). The Reeds-Shepp car is constrained to move with a unit speed (forwards or backwards) and turn with a fixed radius. Although these dynamics are different from those discussed in Section 5.4, we can still define gDPRM with respect to [Schmerling et al. 2015a] and evaluate the benefits of deterministic low-dispersion sampling. Again, spreading was used in the spatial dimension. With the addition of kinodynamic constraints, the Halton and lattice sampling continue to outperform i.i.d. random sampling, as summarized in Table 1 .

\subsection{Summary of Results}

Table 1 shows a summary of the results from simulations detailed in Section 6.3 . Results are shown normalized by the i.i.d. sampling results. In each case the sample count at which a success rate greater than $90 \%$ is achieved and sustained is reported. Additionally, the solution costs at a medium and high sampling count are shown. For all cases the lattice sampling finds a solution with fewer or an equal number of samples and of lower or equal cost than that found by i.i.d. sampling. The Halton sampling also always finds a solution at lower sample counts than i.i.d. sampling, and almost always finds solutions 


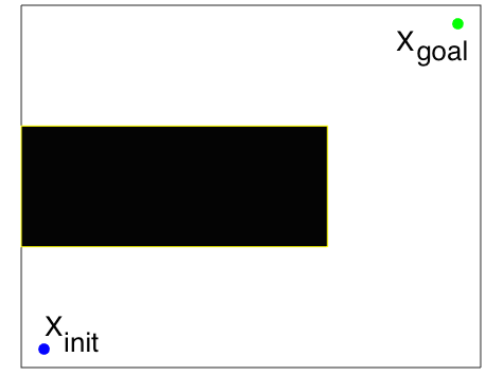

(a)

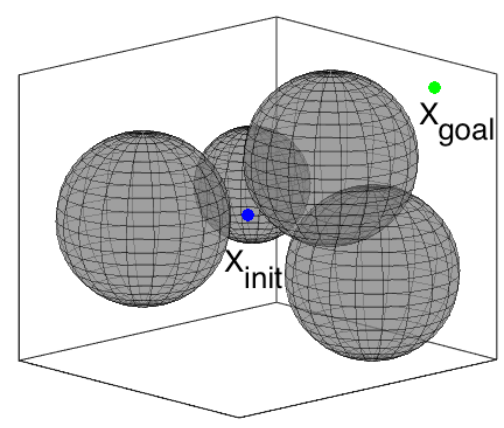

(d)

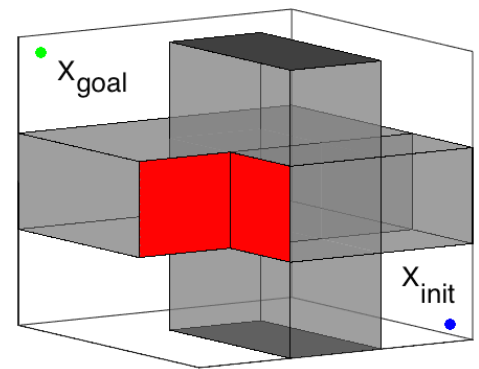

(b)

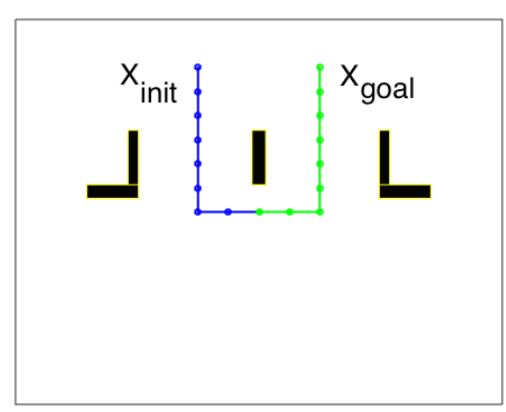

(e)

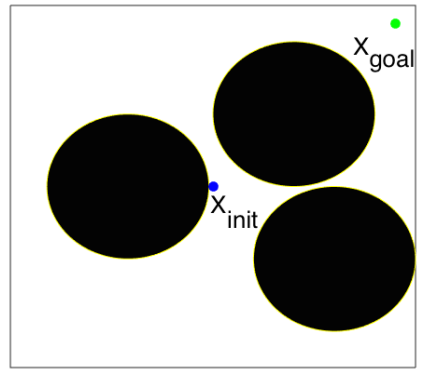

(c)

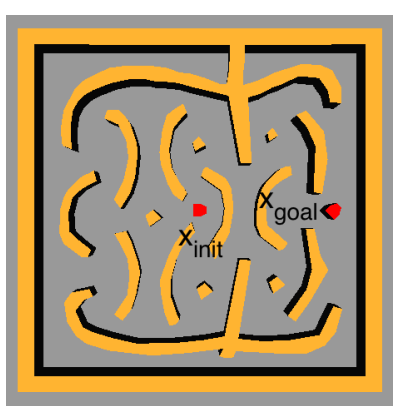

(f)

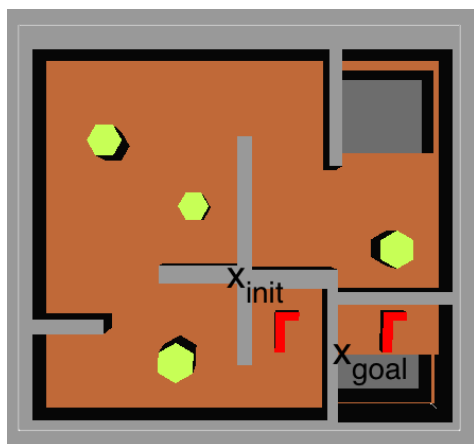

(g)

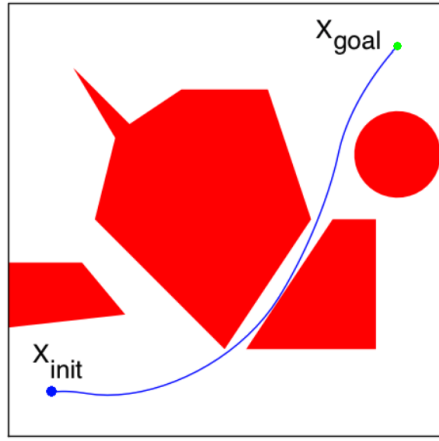

(h)

Fig. 6. Images of the recursive maze planning problem in $2 \mathrm{D} 6(\mathrm{6})$ and $3 \mathrm{D}$ 6(b) and the spherical obstacle sets in $2 \mathrm{D}$ 6(c) and $3 \mathrm{D}$ 6(d). Also shown are an 8D kinematic chain planning problem in 6(e) and the OMPL rigid body planning problems for SE(2) and $\mathrm{SE}(3)$ in 6(f) and 6(g) respectively. Lastly, 6(h) shows the setup for the double-integrator and Reeds-Shepp car. Note that the problems in 6(h) have kinodynamic constraints, where we are not only considering straight line connections, but ones with curvature. A summary of results can be found in Table 1 .

of lower cost as well. The deterministic low-dispersion sequences particularly outperform random sampling in terms of number of samples required for a $90 \%$ success rate.

\subsection{Nondeterministic Sampling Sequences}

The above simulations showed deterministic lattice sampling, with a fixed rotation around each axis, and the deterministic Halton sequence outperform uniform i.i.d. sampling. Both deterministic sequences have low $\ell_{2}$-dispersions of $O\left(n^{-1 / d}\right)$, but sequences with the same order $\ell_{2}$-dispersion need not be deterministic. Figure 7 shows results for a randomly rotated and randomly offset version of the lattice (again, the $\ell_{2}$-dispersion and neighborhoods are all still deterministically the same). The same cases in Table 1 were run for the randomly rotated lattice and the results showed it performed as well as or 


\begin{tabular}{|c|c|c|c|c|c|c|c|}
\hline & & \multicolumn{3}{|c|}{ Halton } & \multicolumn{3}{|c|}{ Lattice } \\
\hline Dim & Obstacles & $90 \%$ Success & Medium & High & $90 \%$ Success & Medium & High \\
\hline 2 & Rectangular & $38 \%$ & $118 \%$ & $80 \%$ & $15 \%$ & $56 \%$ & $80 \%$ \\
\hline 3 & Rectangular & $36 \%$ & $88 \%$ & $94 \%$ & $19 \%$ & $80 \%$ & $87 \%$ \\
\hline 2 & Rect Maze & $13 \%$ & $98 \%$ & $99 \%$ & $13 \%$ & $100 \%$ & $99 \%$ \\
\hline 2 & Sphere & $16 \%$ & $93 \%$ & $99 \%$ & $7 \%$ & $93 \%$ & $99 \%$ \\
\hline 3 & Sphere & $36 \%$ & $97 \%$ & $100 \%$ & $8 \%$ & $97 \%$ & $99 \%$ \\
\hline 4 & Sphere & $100 \%$ & $97 \%$ & $97 \%$ & $100 \%$ & $97 \%$ & $100 \%$ \\
\hline 2 & Recursive Maze & $33 \%$ & $100 \%$ & $100 \%$ & $18 \%$ & $100 \%$ & $100 \%$ \\
\hline 3 & Recursive Maze & $22 \%$ & $95 \%$ & $99 \%$ & $22 \%$ & $96 \%$ & $98 \%$ \\
\hline 4 & Recursive Maze & $56 \%$ & $95 \%$ & $98 \%$ & $56 \%$ & $100 \%$ & $100 \%$ \\
\hline 5 & Recursive Maze & $45 \%$ & $97 \%$ & $96 \%$ & $60 \%$ & $95 \%$ & $96 \%$ \\
\hline 6 & Recursive Maze & $56 \%$ & $95 \%$ & $97 \%$ & $75 \%$ & $94 \%$ & $96 \%$ \\
\hline 8 & Recursive Maze & $56 \%$ & $98 \%$ & $99 \%$ & $75 \%$ & $99 \%$ & $99 \%$ \\
\hline 8 & Chain & $67 \%$ & $112 \%$ & $91 \%$ & $7 \%$ & $76 \%$ & $87 \%$ \\
\hline 3 & $\mathrm{SE}(2)$ & $81 \%$ & $96 \%$ & $100 \%$ & $81 \%$ & $101 \%$ & $101 \%$ \\
\hline 6 & $\mathrm{SE}(3)$ & $32 \%$ & $96 \%$ & $93 \%$ & $42 \%$ & $94 \%$ & $95 \%$ \\
\hline 4 & Double-Integrator & $53 \%$ & $90 \%$ & $93 \%$ & $30 \%$ & $92 \%$ & $96 \%$ \\
\hline 3 & Reeds-Shepp Car & $44 \%$ & $97 \%$ & $99 \%$ & $44 \%$ & $98 \%$ & $100 \%$ \\
\hline
\end{tabular}

Table 1. Summary of results. Each entry is divided by the results of i.i.d. sampling (averaged over 50 runs). For Halton sampling and lattice sampling, the number of samples at which $90 \%$ success is achieved and the cost at a medium number of samples (near 700) and a high number of samples are shown (highest samples simulated, always 3000 or greater). Note that nearly all table entries are below $100 \%$, meaning the Halton and lattice sampling outperformed i.i.d. sampling.

better than random sampling (over 50 runs). In general, low-dispersion random sequences might provide some advantages, e.g., eliminating axis alignment issues while still enjoying deterministic guarantees (see Section 4). Their further study represents an interesting direction for future research.

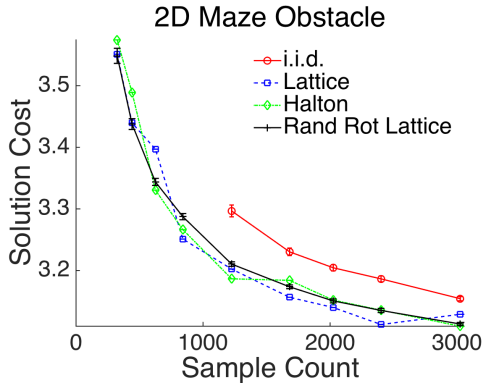

(a)

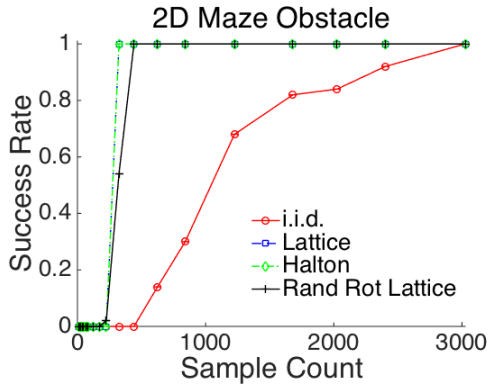

(b)

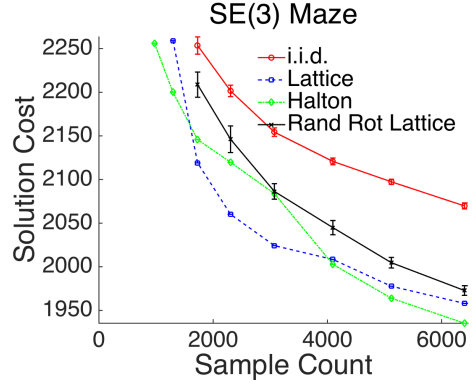

(c)

Fig. 7. Results for deterministic and nondeterministic low-dispersion sampling. "Rand Rot Lattice" refers to a randomly rotated lattice.

\section{Conclusions}

This paper has shown that using low-dispersion sampling strategies (in particular, deterministic) can provide substantial benefits for solving the optimal path planning problem with sampling-based algorithms, in terms of deterministic performance guarantees, reduced computational complexity per given number of samples, and superior practical performance.

This paper opens several directions for future research. First, we plan to deepen our study of deterministic kinodynamic motion planning, in particular in terms of tailored notions of sampling sequences and dispersion and more general dynamical models. Second, it is of interest to extend the results herein to other classes of sampling-based motion planning algorithms (beyond the ones studied in this paper), especially the large class of anytime algorithms (e.g., RRT/RRT*). This leads directly 
into a third key direction, which is to study alternative low-dispersion sampling strategies beyond the few considered here, particularly incremental sequences for use in anytime algorithms. There is already some work in this area, although thus far it has focused on the use of such sequences for the feasibility problem [Yershova and LaValle, 2004, Lindemann et al. 2005. Yershova et al. 2010]. It may also be of interest to study low-dispersion sampling strategies that incorporate prior knowledge of the problem by sampling non-uniformly, as discussed in Section 5.3 . Fourth, we plan to investigate the topological relationship between the optimal path cost and that of the best strong- $\delta$-clear path, in order to frame the convergence rate in terms of the true optimal cost. Fifth, from a practical standpoint, it is of interest to adapt existing algorithms or design new ones that explicitly leverage the structure of low-dispersion sequences (e.g., fast nearest neighbor indexing or precomputed data structures). This would be especially beneficial in the domain of kinodynamic motion planning. Finally, leveraging our convergence rate results, we plan to further investigate the issue of certification for sampling-based planners, e.g., in the context of trajectory planning for drones or self-driving cars.

\section{Acknowledgements}

This work was supported by NASA under the Space Technology Research Grants Program, Grant NNX12AQ43G. Lucas Janson was partially supported by NIH training grant T32GM096982. Brian Ichter was supported by the Department of Defense (DoD) through the National Defense Science \& Engineering Graduate Fellowship (NDSEG) Program.

\section{References}

R. Alterovitz, S. Patil, and A. Derbakova. Rapidly-exploring roadmaps: Weighing exploration vs. refinement in optimal motion planning. In Proc. IEEE Conf. on Robotics and Automation, pages 3706-3712, 2011.

O. Arslan and P. Tsiotras. Use of Relaxation Methods in Sampling-Based Algorithms for Optimal Motion Planning. In Proc. IEEE Conf. on Robotics and Automation, pages 2421-2428, Karlsruhe, Germany, May 2013.

J. Barraquand, L. Kavraki, R. Motwani, J.-C. Latombe, T.-Y. Li, and P. Raghavan. A random sampling scheme for path planning. In International Journal of Robotics Research, pages 249-264. Springer, 2000.

J. Bezanson, S. Karpinski, V. B. Shah, and A. Edelman. Julia: A Fast Dynamic Language for Technical Computing. 2012. Available at http://arxiv.org/abs/1209.5145.

R. Bohlin and L. Kavraki. Path Planning Using Lazy PRM. In Proc. IEEE Conf. on Robotics and Automation, pages 521-528, 2000.

V. Boor, M. H. Overmars, and A. F. van der Stappen. The Gaussian sampling strategy for probabilistic roadmap planners. In Proc. IEEE Conf. on Robotics and Automation, volume 2, pages 1018 - 1023, Detroit, MI, May 1999.

M. S. Branicky, S. M. LaValle, K. Olson, and L. Yang. Quasi-randomized path planning. In Proc. IEEE Conf. on Robotics and Automation, volume 2, pages 1481-1487, 2001.

C.-T. Chen. Linear system theory and design. Oxford University Press, 1995.

I. A. Şucan, M. Moll, and L. E. Kavraki. The Open Motion Planning Library. IEEE Robotics and Automation Magazine, 19(4):72-82, 2012.

P. Deheuvels. Strong bounds for multidimensional spacings. Zeitschrift für Wahrscheinlichkeitstheorie und Verwandte Gebiete, 64(4): 411-424, 1983 .

A. Dobson, G. Moustakides, and K. E. Bekris. Geometric Probability Results for Bounding Path Quality in Sampling-Based Roadmaps after Finite Computation. In Proc. IEEE Conf. on Robotics and Automation, 2015.

J. H. Halton. On the efficiency of certain quasirandom sequences of points in evaluating multidimensional integrals. Numerische Mathematik, 2:84-90, 1960.

D. Hsu, J.-C. Latombe, and R. Motwani. Path planning in expansive configuration spaces. International Journal of Computational Geometry \& Applications, 9(4):495-512, 1999. 
D. Hsu, J.-C. Latombe, and H. Kurniawati. On the probabilistic foundations of probabilistic roadmap planning. International Journal of Robotics Research, 25(7):627-643, 2006.

L. Janson, E. Schmerling, A. Clark, and M. Pavone. Fast Marching Tree: A Fast Marching Sampling-Based Method for Optimal Motion Planning in Many Dimensions. International Journal of Robotics Research, 34(7):883-921, 2015. .

T. Kailath. Linear Systems. Prentice-Hall, Englewood Cliffs, New Jersey, 1980.

S. Karaman and E. Frazzoli. Sampling-based algorithms for optimal motion planning. International Journal of Robotics Research, 30 (7):846-894, 2011.

L. E. Kavraki, P. Švestka, J. C. Latombe, and M. H. Overmars. Probabilistic Roadmaps for Path Planning in High-Dimensional Spaces. IEEE Transactions on Robotics and Automation, 12(4):566-580, 1996.

S. Lavalle. Planning Algorithms. Cambridge University Press, 2006.

S. M. LaValle and J. J. Kuffner. Randomized Kinodynamic Planning. International Journal of Robotics Research, 20(5):378-400, 2001.

S. M. LaValle, M. S. Branicky, and S. R. Lindemann. On the relationship between classical grid search and probabilistic roadmaps. International Journal of Robotics Research, 23(7-8):673-692, 2004.

S. R. Lindemann, A. Yershova, and S. M. LaValle. Incremental grid sampling strategies in robotics. In Workshop on Algorithmic Foundations of Robotics, pages 313-328. 2005.

H. Niederreiter. Random number generation and Quasi-Monte Carlo methods. Number 63 in CBMS-NSF Regional Conference Series in Applied Mathematics. Society for Industrial \& Applied Mathematics, 1992.

J. B. Orlin, K. Madduri, K. Subramani, and M. Williamson. A faster algorithm for the single source shortest path problem with few distinct positive lengths. Journal of Discrete Algorithms, 8(2):189-198, 2010.

J. M. Phillips, N. Bedrossian, and L. E. Kavraki. Guided Expansive Spaces Trees: A Search Strategy for Motion- and Cost-Constrained State Spaces. In Proc. IEEE Conf. on Robotics and Automation, volume 4, pages 3968-3973, 2004.

M. Pivtoraiko, R. A. Knepper, and A. Kelly. Differentially Constrained Mobile Robot Motion Planning in State Lattices. Journal of Field Robotics, 26(3):308-333, 2009.

E. Plaku, K. E. Bekris, B. Y. Chen, A. M. Ladd, and L. E. Kavraki. Sampling-based roadmap of trees for parallel motion planning. IEEE Transactions on Robotics, 21(4):597-608, 2005.

J. A. Reeds and L. A. Shepp. Optimal paths for a car that goes both forwards and backwards. Pacific Journal of Mathematics, 145(2): 367-393, 1990.

E. Schmerling, L. Janson, and M. Pavone. Optimal Sampling-Based Motion Planning under Differential Constraints: the Driftless Case. In Proc. IEEE Conf. on Robotics and Automation, pages 2368-2375, $2015 \mathrm{a}$.

E. Schmerling, L. Janson, and M. Pavone. Optimal Sampling-Based Motion Planning under Differential Constraints: the Drift Case with Linear Affine Dynamics. In Proc. IEEE Conf. on Decision and Control, 2015b. In Press.

A. Stentz. Optimal and efficient path planning for unknown and dynamic environments. International Journal of Robotics \& Automation, 10(3):89-100, 1995.

A. G. Sukharev. Optimal strategies of the search for an extremum. USSR Computational Mathematics and Mathematical Physics, 11(4): 119-137, 1971.

S. Thrun, W. Burgard, and D. Fox. Probabilistic Robotics. MIT Press, 2005.

A. Yershova and S. M. LaValle. Deterministic sampling methods for spheres and SO(3). In Proc. IEEE Conf. on Robotics and Automation, volume 4, pages 3974-3980, 2004.

A. Yershova, S. Jain, S. M. Lavalle, and J. C. Mitchell. Generating uniform incremental grids on SO(3) using the Hopf fibration. International Journal of Robotics Research, 29(7):801-812, 2010. 